\title{
Health and Economic Impact of Routine Pediatric Pneumococcal Immunization Programs in Canada: A Retrospective Analysis
}

\author{
Michele R. Wilson • Matthew D. Wasserman • Marie-Claude Breton • \\ Francois Peloquin · Stephanie R. Earnshaw · Cheryl McDade • \\ Heather L. Sings · Raymond A. Farkouh
}

Received: January 7, 2020 / Published online: April 8, 2020

(C) The Author(s) 2020

\section{ABSTRACT}

Objective: A model was developed to estimate the historical impact (including total societal health and economic benefit) of pneumococcal conjugate vaccine (PCV) programs in the overall Canadian population between 2005 and 2015, inclusively.

Methods: Historical incidence of invasive pneumococcal disease (IPD), pneumonia, and acute otitis media (AOM) were obtained from epidemiologic databases supplemented with published and unpublished data. Two scenarios

Digital Features To view digital features for this article go to https://doi.org/10.6084/m9.figshare.11969340.

Electronic supplementary material The online version of this article (https://doi.org/10.1007/s40121020-00294-6) contains supplementary material, which is available to authorized users.

M. R. Wilson · S. R. Earnshaw · C. McDade RTI Health Solutions, Research Triangle Park, NC, USA

M. D. Wasserman $(\bowtie)$

Pfizer, Inc, New York, NY, USA

e-mail: Matt.Wasserman@pfizer.com

M.-C. Breton · F. Peloquin

Pfizer Canada ULC, Kirkland, QC, Canada

H. L. Sings · R. A. Farkouh

Pfizer, Inc, Collegeville, PA, USA were considered: (1) the observed historical incidence from 2005 to 2015 in the setting of PCV use; (2) a hypothetical scenario in which we estimated the number of disease cases assuming no PCV use. Disease cases averted as a result of PCV programs were calculated by subtracting the number of observed historical cases from the number of estimated cases expected in the absence of PCV use.

Results: PCV programs were estimated to have saved 6631 lives and averted 14,990 IPD cases, 735,700 pneumonia episodes, and 3,697,993 AOM episodes. Positive clinical outcomes resulted in total cost savings of CAD $\$ 1.76$ billion over 11 years. Vaccination costs were offset by the direct medical cost savings from fewer cases of IPD, pneumonia, and AOM.

Conclusions: Canadian PCV programs have provided significant health benefits and resulted in a substantial value for money. Net savings achieved over the reviewed period would have provided funding for $\$ 1.76$ billion in other health care costs or public health initiatives. These findings highlight the importance of considering the total value of a vaccination program, rather than vaccine acquisition costs only, when assessing the value of immunization programs.

Keywords: Acute otitis media; Children vaccination; Pneumococcal disease; Pneumococcal vaccination; Pneumonia; Public health impact 


\section{Key Summary Points}

\section{Why carry out this study?}

Pneumococcal vaccinations have been a part of the routine infant vaccination program throughout Canada since 2005.

The objective of this study was to estimate the historical public health and economic impact of infant pneumococcal vaccine programs in Canada between 2005 and 2015.

\section{What was learned from this study?}

PCV programs were estimated to have saved 6631 lives and averted 14,990 IPD cases, 735,700 pneumonia episodes, and 3,697,993 AOM episodes.

Canadian PCV programs have provided significant health benefits and resulted in a substantial value for money and a net savings over the reviewed period of approximately $\$ 1.76$ billion.

\section{INTRODUCTION}

Streptococcus pneumoniae is a gram-positive bacterium with $>90$ serotypes associated with diseases such as acute otitis media (AOM), pneumonia, and invasive pneumococcal disease (IPD), such as bacteremia and meningitis [1]. Although IPD is more severe and has a greater chance of leading to mortality, pneumonia and AOM represent a significant portion of the burden of disease and associated medical costs [2].

The first pneumococcal conjugate vaccine licensed for use in infants and young children in Canada was a 7-valent pneumococcal conjugate vaccine (PCV7) in 2001. In Canada, the National Advisory Committee on Immunization (NACI) makes recommendations for the use of vaccines while individual provinces are responsible for program implementation and funding. PCV7 became a part of the provincial infant immunization programs (IIPs) between 2002 and 2005. After global PCV7 introduction, IPD incidence due to the seven vaccine serotypes $(4,6 \mathrm{~B}, 9 \mathrm{~V}, 14,18 \mathrm{C}, 19 \mathrm{~F}$, and $23 \mathrm{~F})$ was largely eliminated $[3,4]$ but non-PCV7 serotypes began to emerge, particularly serotype 19A [3]. Subsequently, a 10-valent (PCV10; covering serotypes 1,5 , and $7 F$, in addition to the PCV7 serotypes) and a 13-valent (PCV13; covering serotypes 3, 6A, and 19A, in addition to the PCV10 serotypes) vaccines were approved in Canada in 2008 and 2009, respectively. Since 2008, PCV10 or PCV13 has been a part of the routine infant vaccination program in all provinces, with PCV13 being used in most provinces since early 2011.

Since their inclusion in routine IIPs, PCVs have substantially reduced the incidence of IPD, pneumonia, and AOM in Canada, which translated into a reduction in health care resource use [3-7]. Public health budgets are constrained in Canada, with limited funds available for the introduction of new vaccine programs. Consequently, publicly funded programs may be periodically re-evaluated to ensure resources are used efficiently and that programs in place deliver on what is expected.

While cost-effectiveness is a key criterion in the decision to introduce a new vaccination program in a Canadian province, affordability is a separate consideration. In Canada, once a program is recommended by a vaccine technical committee, budget must then be obtained at the ministry level. Health care funding decision makers will consider the cost of program rather than cost-effectiveness. From an health care system and societal perspective, it is critical that policy makers consider the total financial value of a vaccination program, including costs avoided because of prevented disease cases, rather than focusing solely on vaccination costs. To our knowledge, the financial impact of the pneumococcal IIPs for the Canadian society spanning the use of PCV7, PCV10, and PCV13 coverage has not been previously evaluated. The objective of this study was to quantify the total public health and financial benefit to Canada derived from the publicly funded PCV programs. This article does not involve the study of 
human participants or animals performed by any of the authors and as such was not subject to institutional review board approval. Use of the TIBN data was approved by the Research Ethics Boards (REB) of the participating hospitals and permission to use this data for publication was obtained. CIHI data are publicly available and can be used in publications if they are properly referenced as the source of the data.

\section{METHODS}

We developed a model to quantify the historical public health and economic benefit derived from the publicly funded PCV programs in Canada from 2005 to 2015 inclusively. Two scenarios were considered: (1) the observed incidence of pneumococcal disease from 2005 to 2015, reflecting the era of public PCV use; (2) a hypothetical scenario in which we estimated the number of cases of disease that would occur if no PCV program was introduced in Canada (Fig. 1). The time horizon covers the point when $>90 \%$ of Canadians had access to PCV7 IIPs through the most recent year for which complete incidence data were available at the time of the analysis. Details on the assumptions considered in the model can be found in Appendix table S1.

Averted cases were obtained by subtracting the observed cases from the estimated cases expected in the absence of PCV use (Fig. 1). Direct and indirect costs associated with management of the disease cases were assigned to both scenarios. We then estimated the total retrospective net impact using the following formula:

$$
\text { Net Impact }=\left(X_{\text {without }}-X_{\text {with }}\right)-X_{\mathrm{vx}}
$$

where $X_{\text {without }}$ is the estimated costs associated with cases in the context of no PCV use, $X_{\text {with }}$ is the costs of cases observed since the introduction of the vaccination program, and $X_{\mathrm{vx}}$ is the cost of vaccination (vaccine acquisition + administration).

\section{Population}

Analyses were conducted for the entire Canadian population including vaccinated and

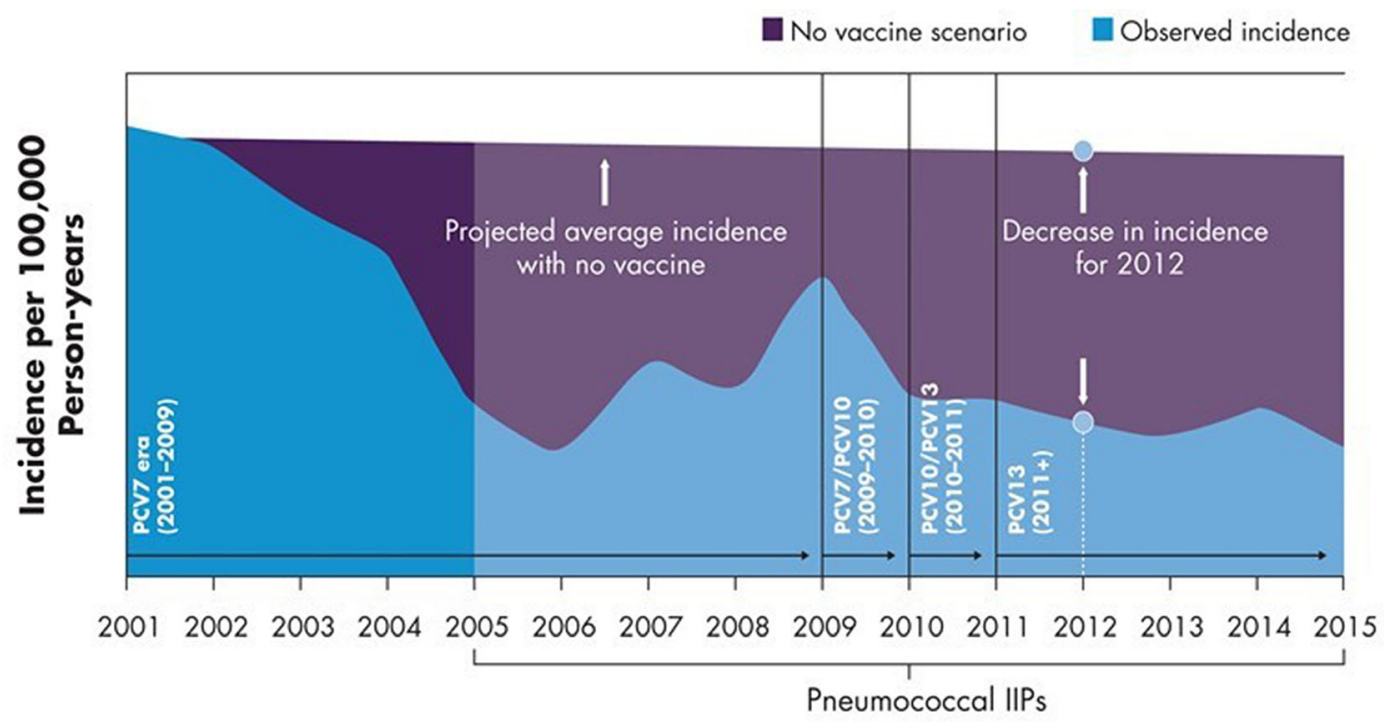

Fig. 1 Model illustration. The figure above illustrates the approach to estimating the historical economic impact of PCV programs in Canada. We take observed incidence from 2001 to 2015 , as represented by the blue area in the graph. The area in blue from 2005 to 2015 represents the observed incidence during the PCV era. To estimate the hypothetical "no PCV program" scenario, we then take incidence prior to implementation of PCV programs (2001-2004) and assume this to be steady-state incidence. The area in purple then represents the estimated cases averted because of PCV programs from 2005 to 2015 
unvaccinated individuals to account for disease reduction as a result of herd immunity. The population was stratified into seven age groups: < 2 years, 2-4 years, 5-17 years, 18-34 years, 35-49 years, 50-64 years, and $65+$ years.

\section{Epidemiologic Data}

IPD incidence was obtained from the Toronto Invasive Bacterial Disease Network (TIBDN), the most robust active, population-based, long-standing surveillance initiative in Canada (Fig. 2). Since there is no active national surveillance program providing serotype-specific incidence or other provincial databases

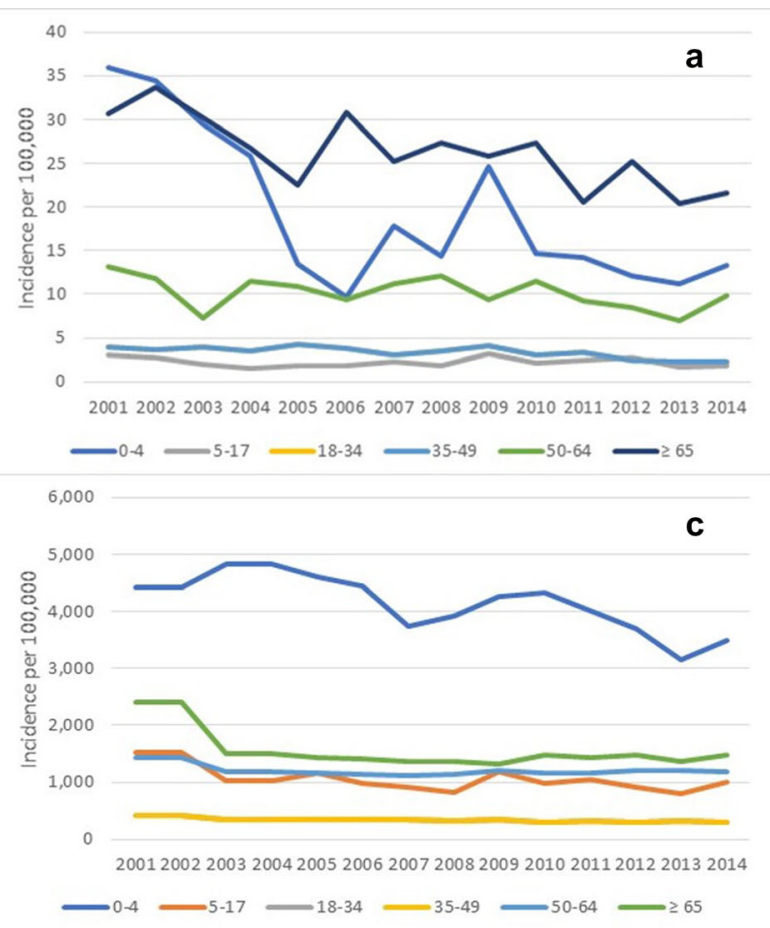

Fig. 2 Historical epidemiologic data (incidence per 100,000), 2001-2015. a Invasive pneumococcal disease incidence per 100,000 by age group, 2001-2015. Disease incidence obtained from TIBDN [41]. b Acute otitis media incidence for ages $<5$ years, 2001-2015. Historical incidence of all-cause AOM is only available from 1996 to 2008 [6]. These data were used to forecast incidence of allcause AOM from 2009 to 2015. The trend in all-cause AOM from 2005 to 2008 showed a similar reduction as seen in the UK following PCV7 introduction. The forecasted incidence of all-cause AOM in 2014 was providing a comparable level of details, TIBDN data were extrapolated to all of Canada. Using all IPD considers historic serotype replacement and reflects the true burden of disease each year without overestimating the reduction by only including vaccine serotypes. The proportion of IPD due to meningitis was obtained from a targeted review of the literature [8] (Table 1).

Hospitalized all-cause pneumonia incidence (ICD10 code J12-J18) was derived from the Canadian Institute for Health Information (CIHI) Discharge Abstract Database for all of Canada, excluding Quebec and British Columbia (2010-2015) as these data were not available [22] (Fig. 2). Cases coded as "most responsible" and "proxy most responsible" as per definition

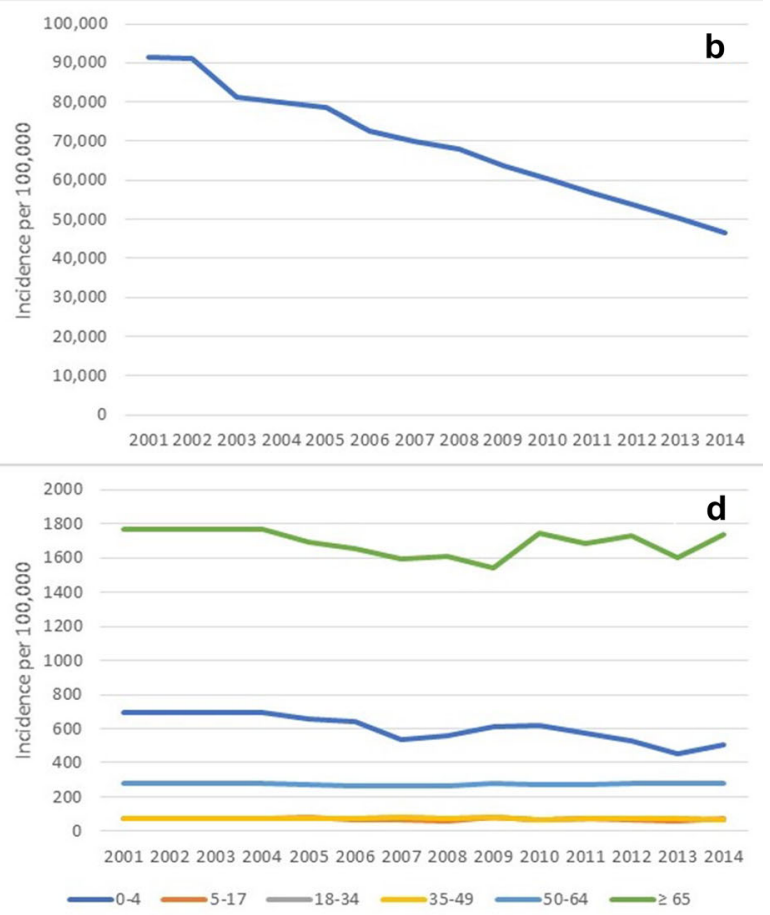

benchmarked against UK observational data to avoid overestimation of impact due to vaccination with PCV13 [24]. Italicized numbers represent forecasted data. Data assumed similar for 2015 as 2014. c Non-hospitalized pneumonia incidence by age group, 2001-2015. Incidence estimated based on the ratio of hospitalized to nonhospitalized pneumonia [42]. d Hospitalized pneumonia: 2004-2014 Canadian Institute for Health Information (CIHI) Discharge Abstract database. Data assumed similar for 2015 as 2014 [22] 
Table 1 Age-specific input parameters

\begin{tabular}{|c|c|c|c|c|c|c|c|}
\hline \multirow[t]{2}{*}{ Parameter (source) } & \multicolumn{7}{|c|}{ Age range (years) } \\
\hline & $<2$ & $2-4$ & $5-17$ & $18-34$ & $35-49$ & $50-64$ & $\geq 65$ \\
\hline Current population [9] & 776,370 & $1,161,631$ & $5,015,400$ & $8,324,245$ & $7,184,090$ & $7,599,967$ & $5,786,907$ \\
\hline $\begin{array}{l}\text { Percentage of IPD presenting as } \\
\text { meningitis [ } 8]\end{array}$ & $9.8 \%$ & $9.8 \%$ & $8.6 \%$ & $5.3 \%$ & $5.3 \%$ & $5.3 \%$ & $3.2 \%$ \\
\hline \multicolumn{8}{|l|}{ Direct costs in Canadian dollars ${ }^{\mathrm{a}, \mathrm{b}}$} \\
\hline Bacteremia $[10]$ & $\$ 19,385$ & $\$ 19,385$ & $\$ 19,385$ & $\$ 33,242$ & $\$ 33,242$ & $\$ 33,242$ & $\$ 23,513$ \\
\hline Meningitis [10] & $\$ 41,348$ & $\$ 41,348$ & $\$ 41,348$ & $\$ 44,198$ & $\$ 44,198$ & $\$ 44,198$ & $\$ 24,137$ \\
\hline Hospitalized pneumonia [10] & $\$ 7356$ & $\$ 7356$ & $\$ 7356$ & $\$ 11,020$ & $\$ 11,020$ & $\$ 11,020$ & $\$ 10,443$ \\
\hline Nonhospitalized pneumonia [8] & $\$ 122$ & $\$ 122$ & $\$ 122$ & $\$ 122$ & $\$ 122$ & $\$ 122$ & $\$ 122$ \\
\hline Acute otitis media $[10]$ & $\$ 170$ & $\$ 170$ & $\$ 170$ & - & - & - & - \\
\hline \multicolumn{8}{|l|}{ Hours of lost productivity per case } \\
\hline Bacteremia $^{c}$ & 61.11 & 61.11 & 61.11 & 88.36 & 90.06 & 90.06 & 82.71 \\
\hline Meningitis $^{c}$ & 99.25 & 99.25 & 99.25 & 120.87 & 122.22 & 122.22 & 79.95 \\
\hline Hospitalized pneumonia $^{c}$ & 39.98 & 39.98 & 39.98 & 52.95 & 53.76 & 53.76 & 62.95 \\
\hline Nonhospitalized pneumonia ${ }^{\mathrm{d}}$ & 6.89 & 6.89 & 6.89 & 4.59 & 4.59 & 4.59 & 4.59 \\
\hline Acute otitis media ${ }^{\mathrm{e}}$ & 6.89 & 6.89 & 6.89 & - & - & - & - \\
\hline \multicolumn{8}{|l|}{ Case fatality rates } \\
\hline Bacteremia [11-17] & 0.012 & 0.012 & 0.012 & 0.078 & 0.078 & 0.12 & 0.291 \\
\hline Meningitis $[11-17]$ & 0.09 & 0.09 & 0.09 & 0.078 & 0.078 & 0.12 & 0.308 \\
\hline Hospitalized pneumonia $[11,18,19]$ & 0.012 & 0.012 & 0.012 & 0.041 & 0.041 & 0.059 & 0.147 \\
\hline
\end{tabular}

IPD invasive pneumococcal disease

a All costs were adjusted to 2018 values

b The health data branch includes all resources used in the hospital but not physician costs, which are paid by the jurisdiction

c Bacteremia, meningitis, and hospitalized pneumonia: lost productivity based on length of stay in the hospital $[10]+$ additional 5 days

d Non-hospitalized pneumonia: 1 work day lost for persons aged 18-64 years, 1.5 work days lost for parents, and 1 day lost for caregivers in persons aged $\geq 65$ years

e Acute otitis media: 1.5 work days lost for parents. Percentage of population participating in the workforce (64.9\%) [20] and average hours worked per week (35.4) $[10,20,21]$

in the Canadian Coding Standards (diagnosis with the longest length of stay and/or resource used during hospitalization) were included [23]. Extraction of data was performed by Chiltern/ OXON [22], and several validation procedures were performed to ensure accuracy and consistency of data and analyses. Non-hospitalized allcause pneumonia incidence was estimated based on the ratio of hospitalized to non-hospitalized pneumonia cases reported [8]. Given that additional factors might have contributed to the observed decline in all-cause pneumonia, we assumed that only $50 \%$ of the observed reductions in incidence were attributable to PCVs.

AOM incidence was obtained from a targeted review of the published literature [6, 24] (Fig. 2). 
Due to a lack of local data after 2008, further reductions ascribed to PCV use were assumed to be comparable to rates observed following PCV7 and PCV13 introduction in the UK [24]. Because no other interventions targeting AOM were introduced during this period, we assumed all differences in AOM incidence to be due to PCV use. This is consistent with a published study showing that substantial changes to both pneumococcal and non-pneumococcal AOM have been observed in countries implementing PCV programs [25].

For the hypothetical scenario with no PCV use, we relied on incidence data as observed in the pre-PCV7 era. For IPD, we assumed a constant disease incidence over the study period based on available pre-PCV7 era incidence data. In Ontario, as reflected in the TIBDN data, IPD incidence began to decline shortly after PCV7 market authorization because of significant uptake in the private market prior to program implementation. By 2002 (1 year post approval by Health Canada), it was estimated that on average at least one dose of PCV7 per newborn was administered [26]. As such, we assumed the incidence of IPD in a setting of no PCV use to be in steady state at the level of 2001 IPD incidence. The baseline for hospitalized pneumonia was estimated from the 2004 all-cause incidence [27]. AOM incidence trends were already declining prior to PCV implementation. As a result, we linearly projected the incidence rate reported by De Wals et al. to estimate AOM incidence in children aged $<5$ years over the study period [6].

\section{Mortality}

IPD and hospitalized pneumonia mortality were obtained from a targeted review of the literature $[11-19,28]$ (Table 1). Occurrence of AOM and non-hospitalized pneumonia was assumed to not impact mortality.

\section{Disease Sequelae}

Conservatively, no sequelae for AOM, IPD, or pneumonia were included in the analysis given uncertainty in the underlying changes in these conditions overtime.

\section{Unit Costs}

Costs included vaccine acquisition and administration, direct medical costs, and indirect disease-related costs and were reported in 2018 Canadian dollars. In Canada, publicly funded vaccines are exclusively contracted with group purchasing organizations under confidential terms. In this study, the confidential contract vaccine price for PCV13 was used as an average for available vaccines over the 11-year period. Negotiated contract prices will vary according to volumes but in general are significantly lower than list prices. Cost of administration was estimated to be $\$ 6.63$ [29]. We assumed that $80 \%$ of infants were vaccinated, based on the national average [30]. We did not consider costs for medical visits as these vaccines are given during routine infant appointments.

Age-specific direct and indirect costs for IPD, pneumonia, and AOM were estimated from the published literature and from the Ontario Case Costing Initiative $[8,10]$ as summarized in Table 1. Indirect costs (hours of lost productivity for patients, parents, and/or caregivers affected by the disease) were estimated by multiplying a duration of illness (as a function of hospitalized length of stay) by the average Canadian hourly wage of $\$ 29.82[10,20,31]$. Details on hours of productivity loss by disease case are shown in Table 1.

\section{Scenario Analyses}

To test the robustness of the model assumptions, we conducted the following series of scenario analyses:

- Using the average IPD incidence from 2001 to 2004 (the period between PCV7 market authorization and 2005 index date) as the annual incidence for the hypothetical scenario with no PCV use (compared with the maximum incidence used in the base case).

- Using the 2004 IPD incidence as the annual incidence for the hypothetical scenario with 
no PCV use (compared with the maximum incidence used in the base case).

- Using 2001 as the annual incidence for the hypothetical scenario with no PCV use.

- Reducing AOM and pneumonia incidence rate decline attributable to PCVs $(50 \%$ and $25 \%$, respectively, compared with $100 \%$ and $50 \%$ in the base case).

- Assuming no incremental impact on AOM incidence after 2008 (the last year of available local AOM incidence data).

- Including AOM cases up to 9 years of age (compared to 5 years in the base case).

- Ninety percent vaccination rates comparable to Quebec (compared to $80 \%$ in base case) $[32,33]$.

- Including Quebec-specific IPD incidence data with Ontario data (TIBDN) (details on Quebec incidence and relative population size can be found in Appendix Figure S1 and Table S2).

- Excluding indirect costs.

\section{RESULTS}

\section{Base Case Results}

Base case results are presented in Table 2. From 2005 to 2015, PCV programs have averted approximately 14,990 cases of IPD, 735,700 cases of pneumonia, 3,697,993 cases of AOM, and 6631 deaths -253 in children $<5$ years old. Reduction in pneumococcal disease burden achieved after the PCV IIPs were translated into net savings for Canada. PCV programs saved a total of $\$ 1.76$ billion over the 11-year period (Table 3, Fig. 3).

\section{Scenario Analysis Results}

Results of scenario analyses are presented in Table 3. PCV programs resulted in net savings over the study period in all scenarios.
Table 2 Base-case results: observed and projected pneumococcal disease cases with and without a PCV IIP, 2005-2015

\begin{tabular}{|c|c|c|c|}
\hline Parameter & $\begin{array}{l}\text { With } \\
\text { PCV IIP }\end{array}$ & $\begin{array}{l}\text { Without } \\
\text { PCV IIP }\end{array}$ & Incremental \\
\hline \multicolumn{4}{|l|}{ Outcomes } \\
\hline \multicolumn{4}{|l|}{ Cases of: } \\
\hline Bacteremia & 26,492 & 40,020 & $-13,528$ \\
\hline Meningitis & 2864 & 4326 & -1462 \\
\hline $\begin{array}{l}\text { Non- } \\
\text { hospitalized } \\
\text { pneumonia }\end{array}$ & $1,765,364$ & $2,448,240$ & $-682,876$ \\
\hline $\begin{array}{l}\text { Hospitalized } \\
\text { pneumonia }\end{array}$ & 690,705 & 743,529 & $-52,824$ \\
\hline $\mathrm{AOM}$ & $12,218,367$ & $15,916,360$ & $-3,697,993$ \\
\hline Deaths & 80,762 & 87,393 & -6631 \\
\hline $\begin{array}{l}\text { Among } \\
\text { those }<5 \text { years }\end{array}$ & 755 & 1008 & -253 \\
\hline $\begin{array}{l}\text { Among } \\
\text { those }>5 \text { years }\end{array}$ & 80,007 & 86,385 & -6377 \\
\hline \multicolumn{4}{|c|}{ Costs (in $\$$ millions) } \\
\hline $\begin{array}{l}\text { Direct medical } \\
\text { costs }\end{array}$ & $\$ 11,001.2$ & $\$ 11,770.8$ & $-\$ 769.5$ \\
\hline $\begin{array}{l}\text { Vaccine-related } \\
\text { costs }\end{array}$ & $\$ 833.9$ & $\$ 0.0$ & $\$ 833.9$ \\
\hline IPD costs & $\$ 810.3$ & $\$ 1200.8$ & $-\$ 390.5$ \\
\hline $\begin{array}{l}\text { Pneumonia } \\
\text { costs }\end{array}$ & $\$ 7286.0$ & $\$ 7872.0$ & $-\$ 586.0$ \\
\hline AOM costs & $\$ 2071.1$ & $\$ 2697.9$ & $-\$ 626.8$ \\
\hline Indirect costs & $\$ 4070.6$ & $\$ 5061.1$ & $-\$ 990.5$ \\
\hline Total costs & $\$ 15,071.9$ & $\$ 16,831.9$ & $-\$ 1760.0$ \\
\hline
\end{tabular}

$A O M$ acute otitis media, IIP infant immunization program, $I P D$ invasive pneumococcal disease, $P C V$ pneumococcal conjugate vaccine

Costs presented in 2018 Canadian dollars 
Table 3 Scenario analyses: total costs (\$ millions)

\begin{tabular}{llll}
\hline Scenario & $\begin{array}{l}\text { With PCV } \\
\text { IIP }\end{array}$ & $\begin{array}{l}\text { Without PCV } \\
\text { IIP }\end{array}$ & Incremental \\
\hline Base case & 15,072 & 16,832 & -1760 \\
Average IPD incidence from 2001 to 2004 used as baseline & 15,072 & 16,668 & -1596 \\
2001 IPD incidence used as baseline & 15,072 & 16,771 & -1700 \\
2004 IPD incidence used as baseline & 15,072 & 16,583 & -1511 \\
Including AOM for 5-9 year olds & 16,572 & 19,182 & -2610 \\
No incremental AOM incidence reduction after 2008 & 15,072 & 15,643 & -571 \\
Only 25\% of pneumonia and 50\% of AOM decline attributed to PCV & 10,686 & 12,055 & -1370 \\
$\quad$ IIP & & & -1656 \\
Vaccination rate set to 90\% for all years & 15,176 & 16,832 & -1809 \\
IPD incidence based on weighted average of Quebec and Ontario data & 15,316 & 17,125 & -770 \\
Exclusion of indirect costs & 11,001 & 11,771 & \\
\hline
\end{tabular}

Costs presented in 2018 Canadian dollars. Base case assumed the following: maximum historical IPD incidence (per 100,000) from 2001 to 2004 for the setting without PCV IIP (35.97 for < 5 years; 3.22 for 5-17 years; 4.29 for 18 -49 years; 13.12 for $50-64$ years; 33.72 for $65+$ years); $80 \%$ vaccination rate for all years; IPD incidence from TIBDN only; incremental reduction in AOM incidence throughout the PCV era; $50 \%$ of pneumonia decline change and $100 \%$ of AOM decline due to PCV IIP introduction; indirect costs considered

For the scenario in which we assume the 2001 incidence for IPD, the incidence rates per age group were: 31.46 for $<5$ years; 3.09 for 5-17 years; 4.00 for $18-49$ years; 13.12 for 50-64 years; 30.70 for $65+$ years

For the scenario in which we assume 2004 incidence for IPD, the incidence rates per age group were: 25.9 for $<5$ years; 1.52 for 5-17 years; 3.48 for $18-49$ years; 11.53 for $50-64$ years; 26.72 for $65+$ years

For the scenario in which we assume the average IPD incidence (per 100,000) from 2001 to 2004, the incidence rates were: 31.46 for $<5$ years; 2.33 for $5-17$ years; 3.78 for $18-49$ years; 10.95 for $50-64$ years; 30.33 for $65+$ years

$A O M$ acute otitis media, IIP infant immunization program, IPD invasive pneumococcal disease, $P C V$ pneumococcal conjugate vaccine

\section{DISCUSSION}

The results of our analysis suggest that the inclusion of PCVs in provincial IIPs in Canada resulted in significant numbers of IPD, pneumonia, and AOM cases averted, which translated into savings $>\$ 1.76$ billion over an 11-year period. This represents approximately $\$ 160$ million savings annually, although interpretation of "annual" results must be done with caution as vaccination impact is not linear over time: the impact is likely to increase with the number of vaccinated individuals and herd effect in the non-vaccinated population. Cost savings from averted disease cases more than offset the cost of vaccine acquisition and administration. Alone, direct medical costs that would have been necessary to treat the nonaverted IPD and pneumonia cases ( $\$ 977$ million) in the absence of PCV use balanced the costs of vaccination ( $\$ 834$ million). While IPD cost savings were substantial, non-invasive disease (pneumonia and AOM) accounted for more than half of the savings. Considering the cost offsets from reduction in pneumococcal disease, a vaccine cost of up to $\$ 224.90$ per dose would have resulted in a cost-neutral scenario from the societal perspective over the 11-year study period. Although PCV programs allowed net savings (cost of vaccination is lower than costs 


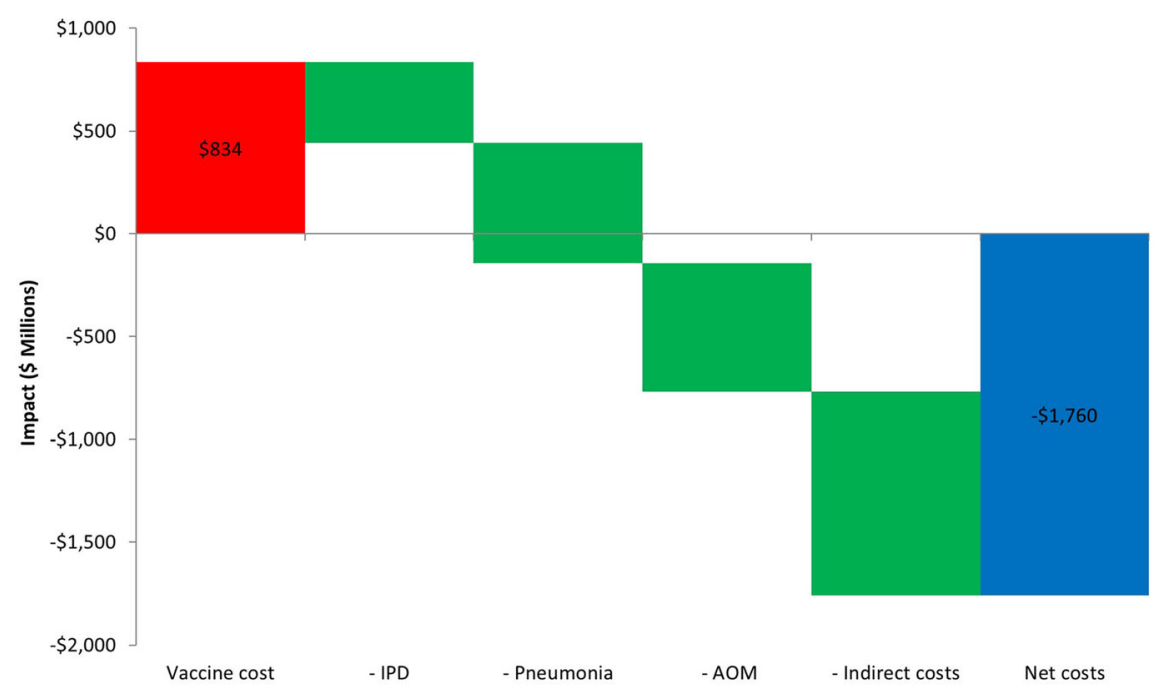

Fig. 3 Economic impact breakdown. The waterfall graph above illustrates the estimated historical economic impact of PCV programs in Canada. The red bar represents the estimated total vaccination cost of PCVs. The subsequent green bars represent the estimated direct medical cost

saved due to averted disease cases) over the time period in all scenarios (Table 3), certain scenarios resulted in smaller overall cost savings.

The estimated net savings may be underestimated, as our assumptions were generally conservative. For example, Luca and colleagues found that incidence of pneumonia hospitalizations and costs have declined by $35-45 \%$ in the PCV era compared with pre-PCV-era data [5]. Our findings suggested a $10 \%$ reduction in pneumococcal pneumonia, substantially lower than that reported in the Luca study. In addition, no costs for sequalae associated with invasive disease were included in the analysis. Hearing loss and neurologic impairment have been estimated to affect approximately $10 \%$ of patients who experience meningitis [8]; as such, our exclusion of any costs related to these sequelae may be conservative.

The literature suggests that by omitting the potential impact on antimicrobial resistance (AMR) in economic models, the true impact of vaccination may be underestimated [34]. In our study, the impact on potential reduction of AMR was not considered. By reducing the incidence of pneumococcal disease, PCV programs may contribute to the reduction of AMR. In savings due to reductions in cases of IPD, pneumonia, and $\mathrm{AOM}$ as well as the indirect costs avoided related to the reductions in cases. The blue bar represents the overall cost savings associated with PCV programs, summing the costs of vaccination with the cost savings from cases averted

fact, following the introduction of PCV13 in Canada, an overall decline in AMR pneumococci has been observed concomitant with the decline in PCV13 serotypes, including the multi-drug resistant serotype 19A [35]. However, modeling AMR is complex, and no reliable data are available on resistance for non-invasive disease. Reduction of prescriptions for antibiotics following implementation of PCV programs is one way to evaluate potential impact on AMR. Based on the number of AOM cases averted in our analysis and assuming that 90\% of all AOM cases in Canada are treated with antibiotics [36], up to 3.3 million antibiotic prescriptions would have been avoided from AOM reduction alone, underlining the value of vaccines as an antimicrobial stewardship tool. This number only accounts for the initial antibiotic prescriptions avoided so the net impact would likely be even larger if subsequent prescriptions due to initial treatment failures were required. A previous analysis provides an example of how this may be implemented within a model [37].

Our study included the impact of disease reduction in the vaccinated and unvaccinated population. The ability of PCVs to confer herd 
immunity is well established [38]. While most of the cost savings in our analysis came from children $<5$ years old, approximately $25 \%$ of the savings were realized in the population aged $\geq 65$ years (results not shown). Failure to include savings stemming from the herd effect would considerably undervalue the benefits of the infant immunization program.

As with any study, this analysis is subject to limitations. For example, due to a lack of nationally representative high-quality surveillance data (programs that collect data on a national basis represent a mixture of different provincial immunization programs), we extrapolated data from an active, populationbased surveillance study from Ontario to all of Canada, assuming that the distribution of cases seen in the TIBDN surveillance data is representative of the general Canadian population. Although IPD epidemiology as well as the implementation and dosing schedule of vaccine programs has varied across provinces, we concluded that extrapolating IPD data from the most robust available surveillance network in Canada was preferable to combining heterogeneous data. Similarly, we extrapolated Ontario disease-specific costs to the rest of the country. The impact of these assumptions on the model results is uncertain, as vaccination coverage, disease transmission risks, and costs may differ in more rural settings. Relatedly, although PCV7 was available in Canada as early as 2001, the implementation of routine PCV7 IIPs occurred at different times across Canadian provinces. We chose 2005 (the year when > $90 \%$ of Canadians had access to PCV7 as part of the IIP) as the anchor year for our analysis. The choice of the analysis start date had no significant impact on results as demonstrated in the scenario analyses.

It is possible that some of the estimated reduction in incidence may be explained by other factors. Examples of such factors could include changes in medical practices, demographics, uptake of the influenza vaccine, etc. Our model was not designed to account for these factors. An additional limitation is that we assumed constant IPD and pneumonia incidence for the scenario in which no PCV was used. It is not possible to know for certain what would have happened in terms of disease incidence without a PCV vaccination program and surveillance data before 2001 were not as good as they were from 2001 onward. Additionally, as noted in the methods section, we did not have complete data for AOM incidence, which required some data assumptions. We also assumed a constant proportion of pneumonia caused by S. pneumoniae. However, PCV programs and use of antibiotics may have changed the disease etiology. Given the limited historical data for pneumococcal pneumonia, it is unclear whether this assumption is correct. Finally, there could be double-counting for cases of invasive hospitalized pneumonia: cases could be captured in the IPD surveillance data as well as in CIHI hospitalization data. It was not possible to differentiate invasive versus non-invasive pneumonia in the CIHI data. The impact it would have on results is uncertain but likely not considerable as the number of invasive disease cases is small compared with cases of non-invasive disease, and we assumed only $50 \%$ of cases of pneumonia would be vaccine preventable.

\section{CONCLUSIONS}

The use of PCV programs has greatly reduced the burden of pneumococcal disease in Canada from 2005 to 2015 . The impact has been seen in reduction of IPD, pneumonia, and AOM as well as approximately 6631 lives saved-253 in children $<5$ years old-in that period. This improvement in clinical outcomes has resulted in a substantial value for money and net cost savings for Canada. These results are only applicable for the study period (2005-2015) and need to be continuously reviewed to account for changing epidemiology and fluctuations during years with high respiratory illness rates and in light of serotype replacement in pneumococcal disease.

When making decisions about funding an immunization program, it is important to consider the net public health value rather than the vaccine acquisition cost only. Estimated net savings of $\$ 1.76$ billion are considerable: for instance, it could be enough to cover the costs 
of $>80,000$ kidney transplants [39] or provide breakfast (at $\$ 2.00$ each) to all elementary school students for two full school years in Canada [40]. This finding reinforces that vaccination provides value for money for the health care system and the society and that it should be prioritized in public health budgets.

\section{ACKNOWLEDGEMENTS}

We acknowledge Dr. Alison McGeer for providing us with the Toronto Invasive Bacterial Disease Network serotype-specific IPD surveillance data and allowing its use for our modeling research. These data were instrumental in our analyses.

Funding. This study was conducted by RTI Health Solutions, Research Triangle Park, NC, under the direction of Pfizer, Inc., and was funded by Pfizer, Inc., New York, NY, which owns Prevnar 13. Pfizer provided funding for the development of the model and manuscript as well as for the rapid service fees. All authors had full access to all the data in this study and take complete responsibility for the integrity of the data and accuracy of the data analysis.

Authorship. All named authors meet the International Committee of Medical Journal Editors (ICMJE) criteria for authorship for this article, take responsibility for the integrity of the work as a whole, and have given their approval for this version to be published.

Disclosures. Michele Wilson, Cheryl McDade, and Stephanie Earnshaw are employees of RTI Health Solutions, who were paid consultants to Pfizer in connection with the development of this manuscript. Matthew Wasserman, Marie-Claude Breton, Francois Peloquin, Heather Sings, and Raymond Farkouh are employees of Pfizer, Inc., who provided funding for the development of this manuscript.

Compliance with Ethics Guidelines. This article does not involve the study of human participants or animals performed by any of the authors and as such was not subject to institutional review board approval. Use of the TIBN data was approved by the Research Ethics Boards (REB) of the participating hospitals and permission to use these data for publication was obtained. CIHI data are publicly available and can be used in publications if it is properly referenced as the source of the data.

Data Availability. This study did not utilize any publicly available datasets. The datasets used for the purpose of the study were generated through custom research requests for the manufacturer and are not publicly available.

Open Access. This article is licensed under a Creative Commons Attribution-NonCommercial 4.0 International License, which permits any non-commercial use, sharing, adaptation, distribution and reproduction in any medium or format, as long as you give appropriate credit to the original author(s) and the source, provide a link to the Creative Commons licence, and indicate if changes were made. The images or other third party material in this article are included in the article's Creative Commons licence, unless indicated otherwise in a credit line to the material. If material is not included in the article's Creative Commons licence and your intended use is not permitted by statutory regulation or exceeds the permitted use, you will need to obtain permission directly from the copyright holder. To view a copy of this licence, visit http:// creativecommons.org/licenses/by-nc/4.0/.

\section{REFERENCES}

1. Public Health Agency of Canada, Streptococcus pneumoniae. Pathogen safety data sheet-infectious substances. 2011.

2. Wilson $\mathrm{M}$, et al. Clinical and economic impact of a potential switch from 13-valent to 10 -valent pneumococcal conjugate infant vaccination in Canada. Infect Dis Ther. 2018;7(3):353-71.

3. Bettinger JA, et al. The effect of routine vaccination on invasive pneumococcal infections in Canadian 
children, Immunization Monitoring Program, Active 2000-2007. Vaccine. 2010;28(9):2130-6.

4. Rudnick W, et al. Pneumococcal vaccination programs and the burden of invasive pneumococcal disease in Ontario, Canada, 1995-2011. Vaccine. 2013;31(49):5863-71.

5. Luca DL, et al. Impact of pneumococcal vaccination on pneumonia hospitalizations and related costs in ontario: a population-based ecological study. Clin Infect Dis. 2018;66(4):541-7.

6. De Wals $\mathrm{P}$, et al. Reduced physician claims for otitis media after implementation of pneumococcal conjugate vaccine program in the province of Quebec, Canada. Pediatr Infect Dis J. 2009;28(9): e271-5.

7. Leal J, et al. Eradication of invasive pneumococcal disease due to the seven-valent pneumococcal conjugate vaccine serotypes in Calgary, Alberta. Pediatr Infect Dis J. 2012;31(9):e169-75.

8. Morrow A, et al. The burden of pneumococcal disease in the Canadian population before routine use of the seven-valent pneumococcal conjugate vaccine. Can J Infect Dis Med Microbiol. 2007;18(2): 121-7.

9. Statistics Canada, Table 17-10-0005-01 (formerly CANSIM Table 051-0001)_Estimates of population, by age group and sex for July 1, Canada, provinces and territories, annual. 2016. https://www150. statcan.gc.ca/t1/tbl1/en/tv.action?pid=1710000501. Accessed Mar 2020.

10. Ontario Health Data Branch Web Portal, Ontario case costing initiative. 2016. Ministry of Health and Long Term Care. https://hsim.health.gov.on.ca/ hdbportal/. Accessed Mar 2020.

11. McNeil, S., et al. Burden of community-acquired pneumonia and invasive pneumococcal disease (Active surveillance for Community Acquired Pneumonia (CAP)) amongst hospitalized Canadian adults, 2011-2013: A Public Health Agency of Canada/Canadian Institutes of Health Research (PCIRN) serious outcomes surveillance (SOS) network study (poster 47) in Canadian Immunization Conference. 2014. Ottawa, Ontario.

12. Smith $\mathrm{KJ}$, et al. Alternative strategies for adult pneumococcal polysaccharide vaccination: a costeffectiveness analysis. Vaccine. 2008;26(11): 1420-31.

13. Statistics Canada, Table 051-0001-Estimates of population, by age group and sex for July 1, Canada, provinces and territories, annual (persons unless otherwise noted). 2013.
14. Centers for Disease Control and Prevention (CDC). National Center for Health Statistics, National Health Interview Survey. 2012. https://www.cdc. gov/nchs/nhis/index.htm?CDC_AA_refVal=https $\%$ 3A\%2F\%2Fwww.cdc.gov\%2Fnchs\%2Fnhis.htm. Accessed Mar 2020.

15. Lexau CA, et al. Changing epidemiology of invasive pneumococcal disease among older adults in the era of pediatric pneumococcal conjugate vaccine. JAMA. 2005;294(16):2043-51.

16. Sadarangani, M., et al. Clinical features and Outcomes of Invasive Pneumococcal Disease in Canada Between 1991 and 2015. in Canadian Immunization Conference. 2018. Ottawa, Ontario, Canada.

17. Atwood M, et al. Cost-effectiveness of alternative strategies for use of 13-valent pneumococcal conjugate vaccine (PCV13) in Canadian adults. Can J Public Health. 2018;109(5-6):756-68.

18. McNeil SA, et al. A Retrospective study of the clinical burden of hospitalized all-cause and pneumococcal pneumonia in Canada. Can Respir J. 2016;2016:3605834.

19. Robinson KA, et al. Epidemiology of invasive Streptococcus pneumoniae infections in the United States, 1995-1998: opportunities for prevention in the conjugate vaccine era. JAMA. 2001;285(13): 1729-35.

20. Statistics Canada, Table 282-0087 and Catalogue no. 71-001-XIE. Labour force characteristics, seasonally adjusted, by province (monthly) (Quebec, Ontario, Manitoba). 2016.

21. Statistics Canada, Table 282-0167-Labour force survey estimates (LFS), average usual hours and wages of employees by age group, sex, union coverage, job permanency, and National Occupational Classification (NOC), unadjusted for seasonality, monthly (persons unless otherwise noted), CANSIM (database). 2017.

22. Canadian Institute for Health Information, Chiltern/OXON report for hospitalized pneumonia: 2004-2014 Canadian Institute for Health Information (CIHI) Discharge Abstract database. Data on file. 2014.

23. Canadian Institute for Health Information. Canadian Coding Standards for Version 2015 ICD-10-CA and CCI. Ottawa: CIHI; 2015.

24. Lau WC, et al. Impact of pneumococcal conjugate vaccines on childhood otitis media in the United Kingdom. Vaccine. 2015;33(39):5072-9.

25. Dagan R, et al. Prevention of early episodes of otitis media by pneumococcal vaccines might reduce 
progression to complex disease. Lancet Infect Dis. 2016;16(4):480-92.

26. McGeer, A., et al. Early impact of PCV10/PCV13 vaccine program on invasive pneumococcal disease (IPD) in Toronto, Canada. Poster \#G3-781a. In: Interscience Conference on Antimicrobial Agents and Chemotherapy. Chicago IL, USA, 2011.

27. Tsaban G, Ben-Shimol S. Indirect (herd) protection, following pneumococcal conjugated vaccines introduction: a systematic review of the literature. Vaccine. 2017;35(22):2882-91.

28. McNeil, S. and et al. Burden of community acquired pneumonia and invasive pneumococcal disease amongst hospitalized Canadian adults: A Public Health Agency of Canada/Canadian Institutes of Health Research (PCIRN) serious outcomes surveillance network study. In: PCIRN Annual Meeting. 2014. Toronto, Ontario, 2014.

29. De Wals $\mathrm{P}$, et al. Cost-effectiveness of immunization strategies for the control of serogroup C meningococcal disease. Vaccine. 2004;22(9-10): 1233-40.

30. Government of Canada, Vaccine uptake in Canadian children: Highlights from childhood National Immunization Coverage Survey. 2015.

31. Statistics Canada, Table 282-0225-Labour force survey estimates (LFS), average weekly earnings, average hourly wage rate and average usual weekly hours by union status and type of work, Canada and provinces, annual. 2016.

32. Comité sur l'immunisation du Québec, Avis sur le calendrier optimal de vaccination des enfants contre les infections à pneumocoque au Québec September 2017, l'Institut national de santé publique du Québec (INSPQ). 2017.

33. Kiely, M., et al., Enquête sur la couverture vaccinale des enfants de 1 an et 2 ans au Québec en 2016. Québec (QC): Institut national de santé publique, 2018. Disponible à. 2018.
34. Atkins $\mathrm{KE}$, Flasche $\mathrm{S}$. Vaccination to reduce antimicrobial resistance. Lancet Glob Health. 2018;6(3):e252.

35. Public Health Agency of Canada, Canadian antimicrobial resistance surveillance system-report. 2016. https://www.canada.ca/en/publicheal th/services/publications/drugs-health-products/can adian-antimicrobial-resistance-surveillance-systemreport-2016.html. Accessed Mar 2020.

36. Quach C, Collet JP, LeLorier J. Acute otitis media in children: a retrospective analysis of physician prescribing patterns. Br J Clin Pharmacol. 2004;57(4): $500-5$.

37. Breton $\mathrm{M}$, et al. Modeling reductions in antibiotic prescriptions for otitis media as a result of pneumococcal conjugate vaccination in Canada in IDWeek 2018. San Diego: IDWeek; 2018.

38. Public Health Agency of Canada, Canada Communicable Disease Report: An Advisory Committee Statement (ACS) National Advisory Committee on Immunization (NACI) update on the use of pneumococcal vaccines in adults 65 years of age and older-a public health perspective 2018 .

39. Canadian Institute for Health Information, Patient Cost Estimator. 2017. https://www.cihi.ca/en/ patient-cost-estimator. Accessed Mar 2020.

40. Breakfast Club of Canada, 1 in 4 Canadian children might not eat breakfast today. 2020. https://www. breakfastclubcanada.org/. Accessed Mar 2020.

41. TIBDN, Toronto Invasive Bacterial Diseases Network. Department of Microbiology, Mount Sinai Hospital, Toronto, ON, Canada, 2016.

42. Nelson JC, et al. Impact of the introduction of pneumococcal conjugate vaccine on rates of community acquired pneumonia in children and adults. Vaccine. 2008;26(38):4947-54. 